\title{
Removal of Pectoral Muscle in Mammograms using Statistical Parameters
}

\author{
Sonali Bhadoria \\ Asst. Prof, \\ MAE, Pune
}

Yash Bharwani Anikta Pati

\begin{abstract}
The image segmentation is the basic step in the detection of tumors in various medical images. Specially when used for CAD system. Presence of pectoral muscles gives very false results in the detection process. Removing pectoral muscles is a very important issue in Mammograms. This paper address this issues of Pectorial muscles removal from the mammogram image. We have extracted various features of the mammogram images and their ranges to remove the unwanted part of pectoral muscles which remains even after the segmentation. This method is very simple and yet very effective to achieve the exact ROI.
\end{abstract}

\section{General Terms}

Segmentation; Mammogram; Thresholding; Pectoral muscle;

\section{INTRODUCTION}

Breast cancer is the leading cause of cancer deaths among women in many countries. Breast cancer incidence and death rates generally increases with age according to studies.. During $2000-2004,95 \%$ of new cases and $97 \%$ of breast cancer deaths occurred in women aged 40 and older [1],[2]. Amongst the many imaging techniques available for breast cancer detection mammography is considered as the most effective method.

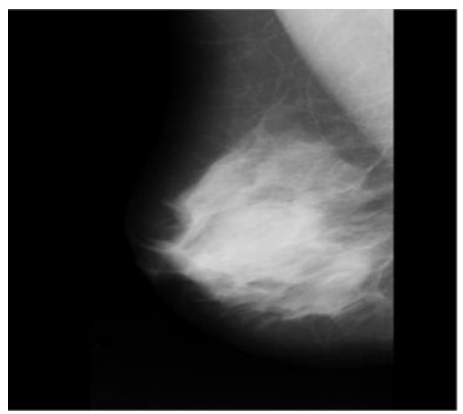

Fig 1: Example of a mammogram

. Fig 1 shows an example of a mammogram which is the x-ray image of the breast. Image segmentation is an very first step in processing the mammogram image for further analysis. [3]. Segmentation of mammograms can be done in two basic steps. In the first step we aim to remove the unwanted breast part and in the second we try to remove the pectoral muscles. This gives us the exact ROI. In the Computer Aided Detection (CAD) of breast cancer we need to segment the mammographic images into various texture regions representing different tissue types. The main objective behind the segmentation of the medical image is to separate the tumor from the background. [4]. The increase in size of medical image database, has led to the use of computers in facilitating their processing and analysis. Estimation of the volume of the whole organ, parts of the organ and/or objects within an organ i.e. tumors is important step in the analysis of medical image [5]. Majority of early breast cancer can be diagnosed by detecting micro calcification clusters in mammographic $\mathrm{X}$-ray images. The micro calcifications appear in small clusters of few pixels with relatively high intensity compared with their neighboring pixels. They are extremely minute and elongated salt-like particles and are sometimes no larger than $0.1 \mathrm{~mm}$ in size. They are responsible for detection of $43-49 \%$ of all cancer detected through mammogram [6]. Various image features can be extracted and preserved by a detection system. Characteristics of these features in the original play a key role in differentiating various organs. [7]. Detecting micro calcifications is difficult as they are embedded in a nonhomogeneous background. [8], [9].

\section{ISSUES IN MAMMOGRAM \\ SEGEMENTATION}

There are various problems which we face during the segmentation as mentioned here. Raw digital mammograms are medical images that are difficult to interpret [10]. Lesser radiation dose of mammographic images degrades the contrast and the overall visibility of the micro calcifications and tumorous mass regions from the surrounding tissue [5]. Due to presence of edges of low signal-to-noise ratio and complicated structured background, detection of a subtle mass on a mammogram is a difficult task [11]. There is a problem in segmentation of mammogram to a simple fatty and non-fatty set of regions due to large differences in parenchymal type appearances and variability of image acquisition parameters [12]. Pectoral muscle detection is a challenging task because it is not very well differenced from the surrounding breast tissue. There is very small intensity variation of the pectoral muscle and the tumor tissue for each mammogram images. Due to the presence of pectoral muscle detection procedures gets biased, and hence should be removed during mammogram pre-processing [13].Fig 2 shows the locally thresholded image in which pectoral muscle can be seen in the 3rd tile of 1 st row.
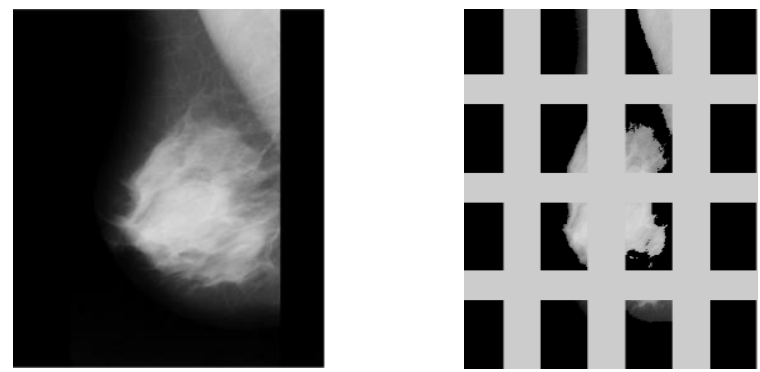

Fig 2:- (a)original image; (b)Locally segmented image 


\section{ISSUES IN REMOVING PECTORAL MUSCLE}

Pectoral muscles are the regions in mammograms that contain brightest pixels. These regions must be removed before detecting the tumor cells so that mass detection can be done efficiently. Pectoral muscles lie on the left or right top corner depending on the view of the image. We must detect the position of the pectoral muscles (left top corner or right top corner) before removing it [14].

Extracting the pectoral muscle $[15,16,17]$ is particularly important in automated mammogram image assessment. Segmentation of the pectoral muscle is a non-trivial, complex and demanding task. It is also complicated due to a number of factors. Firstly, the muscle edge is not a straight line, but can be convex, concave or a mixture of both. Secondly, muscle edge though may appear to be visually continuous; the edge exhibits variations in texture and sharpness [18].

\section{EARLIER METHODS USED}

There have been various techniques to remove the pectoral muscle and isolate the breast region. M. Wirth et al. developed an algorithm that uses morphological preprocessing and fuzzy rule-based algorithm for breast region extraction. Kostas Marias et al. used the boundary extraction technique based on a combination of the Hough transform followed by image gradient operators and morphology in order to make coherent the breast region part of the image. Histogram equalization and thresholding process are employed by Barba J. Leiner et al. to extract only the region of the image that corresponds to the breast. Segmentation of the breast region in mammograms has traditionally been achieved using methods besides active contours. Semmlow et al. used a spatial filter and Sobel edge detector to locate the breast boundary on xeromammograms. Global thresholding has been used in many cases to segment the breast region from the background. The major problem with using global thresholding is the nonuniform background region, although efforts, such as that of Masek et al. using local thresholding have shown more promise. A system of masking images with different thresholds to find the breast edge is developed by Abdel-Mottaleb et al.Gradient based method is proposed by Méndez et al. to find the breast contour. They used a two level thresholding technique to isolate the breast region of the mammogram [18].

\section{PROPOSED ALGORITHM}

In order to remove the Pectoral muscle following steps were used.

i) The mammogram image is transformed into number of equal tiles.[19]

ii) Adaptive thresholding technique is used to segment the image by obtaining threshold, individually for each tile. Instead of finding the global threshold value and then segmenting the image, we have used local processing method. By finding out the local statistics of every tile, we get better segmentation results. Fig 3 is mbd010 shows the difference in local and global thresholding.

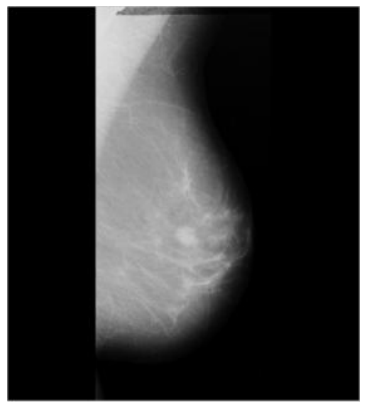

(a)

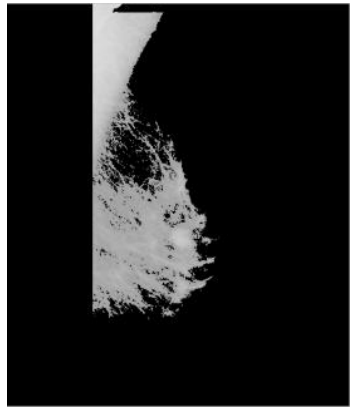

(b)

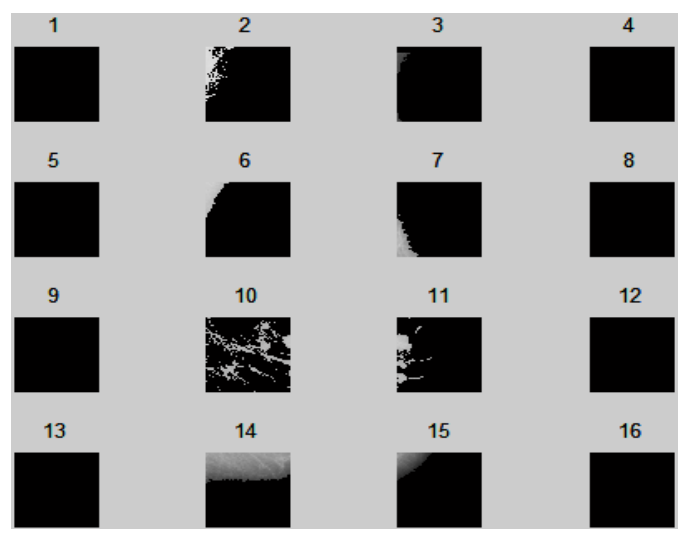

(c)

Fig 3:- (a) Original image; (b) Globally thresholded image;(c) Locally thresholded image.

iii) Various statistical features were extracted from each tile and their properties were analyzed. It has been observed that there were different ranges of the features for tumor region and pectoral muscles as shown in fig 4 .

iv) After studying the ranges of these distinct features filters were designed to eliminate the pectoral muscles. It gave us good removal of the unwanted rerion and exact ROI was extracted.

\section{RESULTS}

The input images, on which we have tested the algorithm, have been taken from MIAS database. Fig 4 (a), (b) and (c) shows the plot of Standard deviation of image, mean of image and standard deviation of the histogram for pectoral muscle and tumor part. These different ranges shown were used to design the filters. Fig 5(a) is showing a locally thresholded benign image mdb001.pgm which contains pectoral muscle(see tiles indicated with an arrow) and (b) is showing a locally thresholded benign image mdb001.pgm from which pectoral muscle has been removed (see tiles 4 indicated with an arrow). 


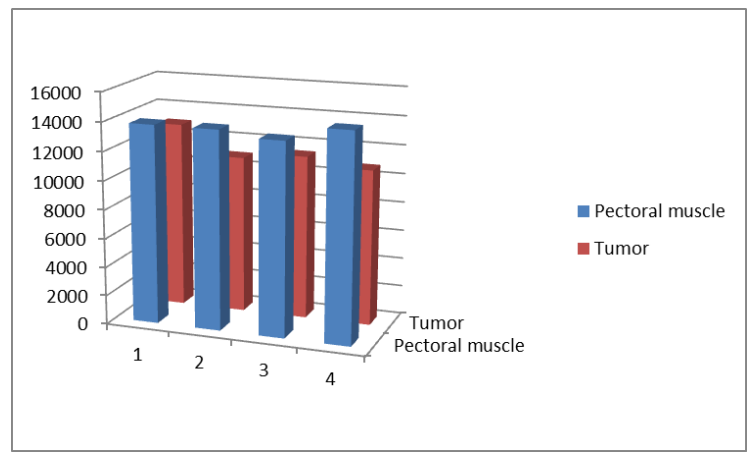

(a)

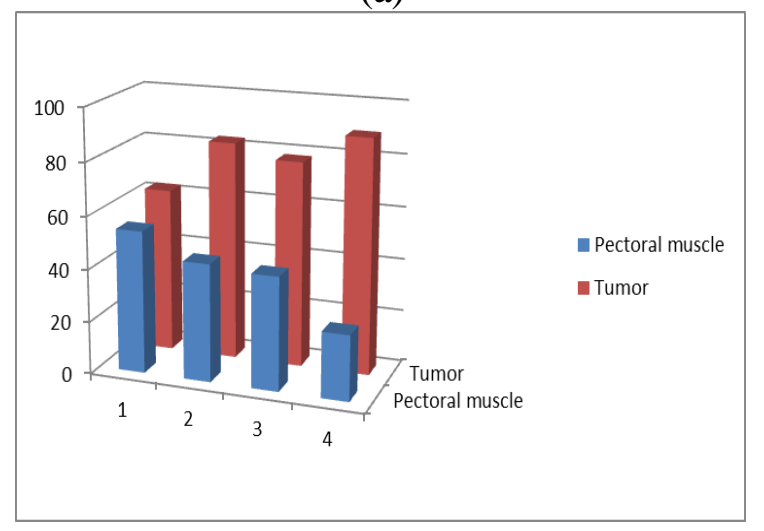

(b)

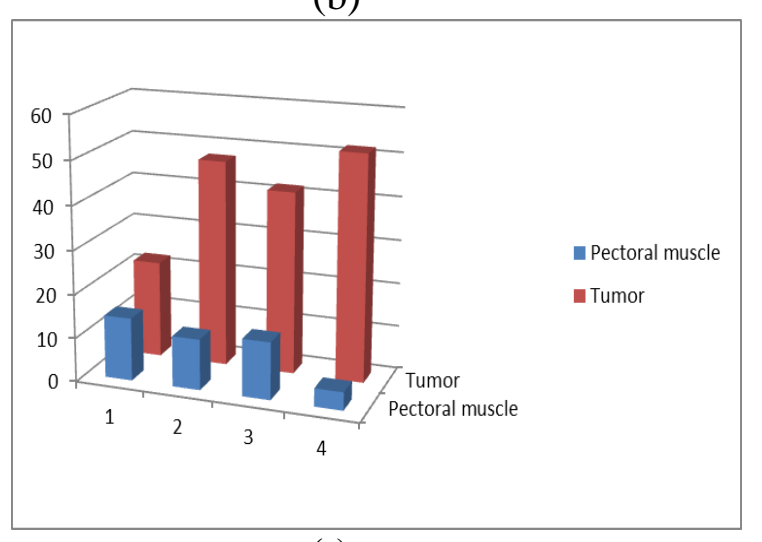

(c)

Fig 4: (a)Graph displaying the standard deviation of pectoral muscle and tumor region; (b) Graph displaying the mean of pectoral muscle and tumor region; (c) Graph displaying the standard deviation of histogram of pectoral muscle and tumor region;

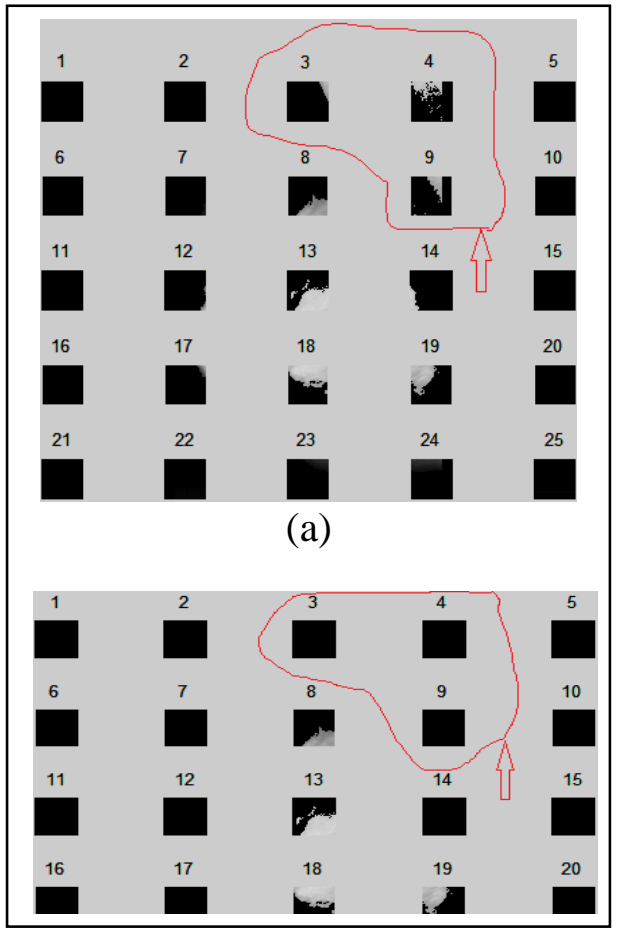

Fig 5: (a)Image with pectoral muscle; (b)image without pectoral muscle

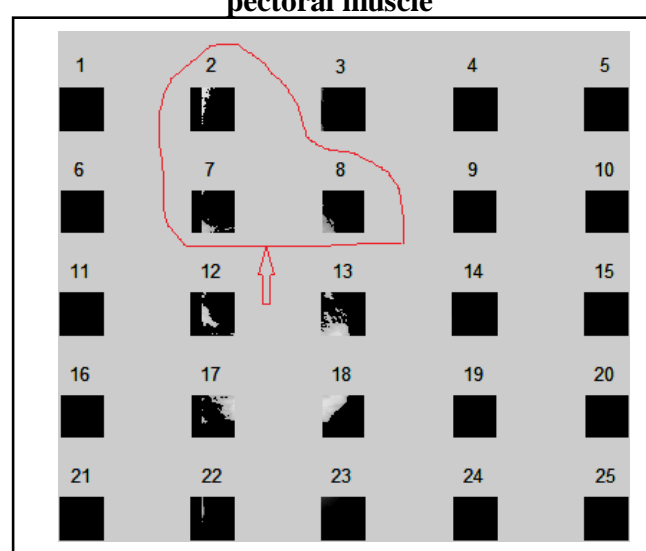

(a)

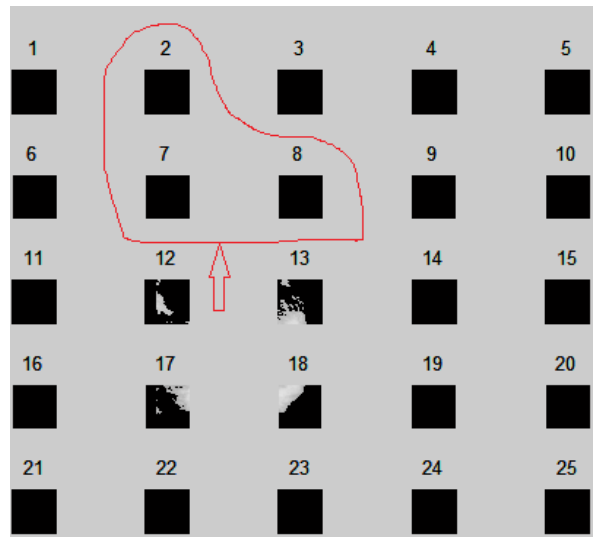

(b)

Fig 7:- (a) Image with pectoral muscle; (b) image without pectoral muscle

Fig 7 (a) is showing a locally thresholded malignant image mdb072.pgm which contains pectoral muscle(see tile indicated with an arrow) and (b) is showing a locally thresholded 
malignant image mdb072.pgm from which pectoral muscle has been removed (see tile indicated with an arrow).As the limits for malignant are different form benign image, we can see small part of pectoral muscle visible in the final result.

\section{CONCLUSION}

After undergoing various tests it can be concluded that Standard deviation of histogram is unique feature to find out the difference in pectoral muscle and tumor region.However it is very difficult to find out one statistical limit which increases the true negative rate for all different classes of mammogram images. To improve the true negative rate, more statistical features can be added.

\section{REFERENCES}

[1] American Cancer Society,Breast Cancer Facts and Figures 2007-2008,Atlanta,Georgia:American Cancer Society ,Inc.2007.

[2] Jaya Sharma \& Sujeet Sharma,"Mammogram Image Segmentation using Watershed", International Journal of Information Technology and Knowledge anagement.

[3] Byung-Woo Hong and Michael Brady," Segmentation of Mammograms in Topographic Approach", Medical Vision Laboratory, University of Oxford, U.K.

[4] Sonali Bhadoria, Preeti Agrawwal, CG Dethe, Renu Vig,' Role of Segmentation in Medical Imaging: A Comparative Study 'International Journal of Computer Applications (0975 - 8887)’

[5] H.B.Kekre and Saylee Gharge " Texture Based Segmentation using Statistical Properties for Mammographic Images",(IJACSA) International Journal of Advanced Computer Scirnce and Apllications,Vol, 1,No.5,November 2010.

[6] C. H. Chen1,2 and G. G. Lee," On Digital Mammogram segmentation and microcalcification detection using multiresolution wavelet analysis",Electrical and Computer Engineering Department, University of Massachusetts Dartmouth, 285 Old Westport Road, N.Dartmouth, Massachusetts 02747.

[7] Wang, T.C. Karayiannis, N.B. "Detection of microcalcifications in digital mammograms using wavelets", Dept. of Electr. \& Comput. Eng., US Robotics, Stokie, IL, USA.

[8] M.Melloul,"Segemntation of Microcalcifications in X-ray Mammograms using Entropy Based Thresholding",Masters Thesis, The Hebrew University of Jerusalem,Dec. 2001. Available in http://www.cs.huji.ac.il/ josko/caspublications.html.

[10] Moti Melloul and Leo Joskowicz,'Segmentation of microcalcification in X-ray mammograms using entropy thresholding",School of Computer Science and Engineering.
[11] Indra Kanta Maitra,Sanjay Nag and Samir K.Bandyopadhyay,'Dtection of Abnormal Masses using Divide and Conquer Algorithm Digital Mammogram",Dept. of Computer Science \& Engineering, University of Calcutta, 92 A.P.C. Road,Kolkata-700009, West Bengal,India.

[12] Dr.H.B.Kekre1, Saylee M. Gharge2 and Tanuja K. Sarode3,'Image Segmentation of Mammographic Images Using Kekre'S Proportionate Error Technique on Probability Images", International Journal of Computer and Electrical Engineering, Vol.2, No.6, December, 201017938163.

[13] Styliani Petroudi and Michael Brady,"Breast Density Segmentation Using Texture",Wolfson Medical Vision Laboratory ,Oxford University,Oxford,OX@ 7DD,United Kingdom.

[14] Nagi, J.; Abdul Kareem, S.; Nagi, F.; Kaleel Ahmed," Automated breast profile segmentation for ROI detection using digital mammograms", S.; Fac. of Comput. Sci. \& Inf. Technol., Univ. of Malaya, Kuala Lumpur, Malaysia.

[15] T.S.Subhashini,V.Ramalingam \&S.Palanivel, "Pectoral Muscle removal and Detection of masses in Digital Mammogram using CCL",IJCA(0975-8887) Volume 1No.6.

[16] K. Thangavel, And M.Karnan,"Computer Aided Diagnosis in Digital Mammograms: Detection of microcalcifications by Meta Heuristic Algorithms", GVIP Journal,Volume 5,Issue 7 July 2005

.[17] S.M Kwok,R chandrashekar, and Y.Attikiouzel,"Automatic Pectoral Muscle Segmentation on Mammograms by Straight Line Estimation and Cliff Detection",7th Australian an New Zealand Intelligent Information Systems Conference 18-21 November 2001,Perth,Western Australia.

[18] H.Mirzaalian,M.R. Ahmedzadeh, and S. Sadri, "Pectoral Muscle Segmentation on Digital Mammograms by nonlinear Diffusion Filtering",1-4244-1190-407/2007 IEEE,pp.581584.

[17] Arun Kumar \& H.S.Sheshadri,"Breast Contour Extraction and Pectoral Muscle Sgementation in Digital mammograms",IJCSIS

[19] Sonali Nakade, P.T. Karule, "Mosaicture: Image Mosaic Generating System Using CBIR Technique," iccima, vol. 3, pp.339-343, 2007 International Conference on Computational Intelligence and Multimedia Applications, 2007DOI: http://doi.ieeecomputersociety.org/10.1109/ICCIMA.2007.1 44 